\title{
Hadrons anti-hadrons production in reaction planes in relativistic heavy ion collisions on two common event generators: HIJING and HYDJET++
}

\author{
M.H.M. Soleiman ${ }^{a}$, S.S. Abdel-Aziz, and M.S.E. Sobhi \\ Cairo University, Faculty of Science, Physics Department, Giza, Egypt
}

\begin{abstract}
The study of hadron production in heavy ion collisions provides the researcher with valuable techniques to investigate the properties of quark gluon plasma (QGP). The hadron and anti-hadron production in the reaction plane, at relativistic heavy ion collisions are studied for the energy per nucleon available at the RHIC-STAR experiment of $\left.\sqrt{(} s_{N N}\right)=200 \mathrm{GeV}$. In order to test the models used for hadron and anti-hadron production, two common event generators (HIJING 1.35 and HYDJET++2.0.2), established on Monte Carlo techniques, are discussed for hadron and anti-hadron production in the reaction plane for heavy ions ${ }^{197} A u_{79}-{ }^{197} A u_{79}$ collisions at $\sqrt{S_{N N}}=200 \mathrm{GeV}$. We find that, HYDJET ++2.0 .2 is more realistic in comparing the results of simulations with those of the experimental data published by the STARCollaboration.
\end{abstract}

\section{Introduction}

Relativistic heavy-ion collisions create suitable conditions for a phase transition from hadron to deconfined quark matter QCD and a test benchmark for lattice QCD calculations. Many evidences have demonstrated that the quark gluon plasma (QGP) matter has been produced mainly in the central $A u-A u$ collisions at RHIC energies [1]. In the process, large amounts of energy are deposited into a more extended volume than that achieved in elementary particle collisions. These nuclear interactions briefly produce hot and dense matter containing roughly equal numbers of quarks and antiquarks. Then the QGP expands rapidly and cools down. QGP undergoes a transition into unstable hadron gas, producing hadrons, anti-hadrons, and even complete nuclei as was reported by the RHIC-STAR experiment - USA in 2010 [2], where the discovery of anti-Helium $4\left({ }^{4} \mathrm{He}_{2}\right.$ or $\left.\alpha\right)$ show the possibility of new dynamics in producing anti hadrons from QGP. Therefore the relativistic heavy-ion collision can not only provide an environment to study strong interacting phase transition and QCD matter but also an ideal venue to produce antimatter particles [3].

There are research proposals due to Kharzeev and Voloshin [4-6] about the rule of following the steps of chiral symmetry breaking: a- Rotation of charged quarks in QGP, b- Self-induced magnetic field, c- Generation of Faraday and Hall currents, d- Chiral flipping of left helicity to right helicity quarks. e- Charge separation due to selfinduced magnetic field.

The global study of the ratio over all generated events is carried out in [7-9]. The event by event asymmetry calculation and then averaging is carried out in this investigation and assigned a convention of a local study.

\footnotetext{
a e-mail: mhm.soleiman@gmail.com; mhmsoleiman@cu.edu.eg
}

The present work investigates the ability of the common models "HIJING 1.35", and "HYDJET++ 2.0.2" used in studying QGP phenomenology with and without collective flow, respectively, by Monte Carlo techniques [3]; to reproduce phase space (i.e. spatial and momentum ) distributions asymmetry in antihadron to hadron ratios. The spatial asymmetry distributions take the reaction plane as a spatial reference in estimating the asymmetries in antihadron to hadron ratios. We restrict our investigation to two baryons and two mesons. That are; proton and lambda $\left(p^{ \pm}, \Lambda^{ \pm}\right)$for baryons, and pi and kaon $\left(p^{ \pm}, \kappa^{ \pm}\right)$for mesons. Our results could be investigated at the relativistic heavy ion collider (RHIC), and applied on the data from the experiments of STAR, PHOBOS, PHENIX, BRAHMS in USA-BNL.

The paper is organized in order to show the reader the possibility to detect charge asymmetry in samples of events generated by each the event generators HIJING 1.35, and HYDJET ++2.0 .2 . The charge asymmetry appear and are detected without regarding the Kharzeev and Voloshin effects of the self-induced global chiral magnetic field and the subsequent charge separation of quarks and anti-quarks, since these effects are not included in any of the studied event generators. After the introduction, Sect. 2 will introduce basic definitions and methods used throughout the discussion. Section 3 gives the results and discussions. Finally Sect. 4 summarizes the conclusions and future work.

\section{Methods and concepts}

Hijing 1.35 is an event generator written in Fortran 77 , based on QCD inspired models for multiple jet production, and designed to study jet and minijet production and associated particle production in $p p, p A$, and $A A$ ultra-relativistic collisions. This 

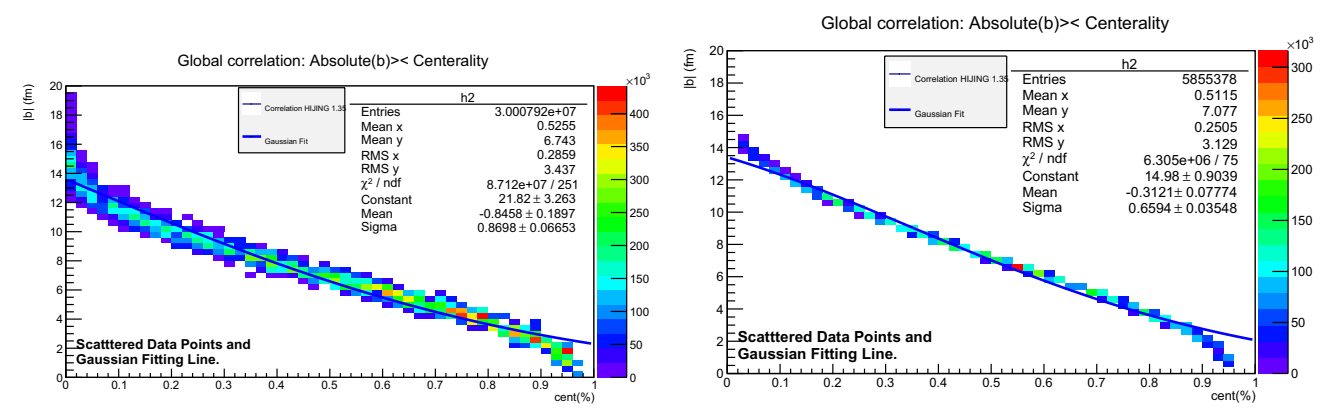

Figure 1. The correlation between the impact parameter $|\mathbf{b}|$ and the centrality. The solid line is the most probable Gaussian fit. The colors represent the densities of points. Graph (a) gives the results obtained on HIJING 1.35, and graph (b) is the results deduced on HYDJET ++ 2.0.2.

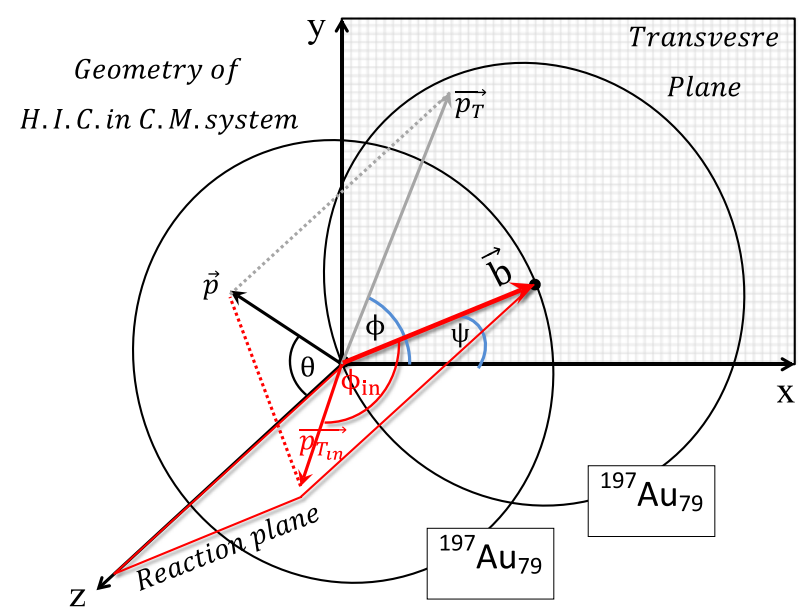

Figure 2. The geometry of heavy ion collision event. The reaction plane is represented in red. The transverse plane is represented as xy-plane.

model incorporates mechanisms such as multiple minijet production, soft excitation, nuclear shadowing of parton distribution functions and jet interactions in dense hadronic matter. Each generated event and the produced particles represents one probable state of a physical system. The flexibility of programming allows for testing several hypothesis and modifications on the dynamical models of QGP. HYDJET ++ 2.0.2. HYDJET ++ event generator [11] includes detailed treatment of soft hadroproduction as well as hard multi-parton production, and takes into account medium-induced parton re-scattering and energy loss. The main program HYDJET ++ is written in the object-oriented $C++$ language under the ROOT environment $[12,13]$. In our study HIJING 1.35 and HYDJET ++2.0 .2 are tuned to ${ }^{197} A u_{79}-{ }^{197} A u_{79}$ relativistic heavy ion collisions at $\sqrt{s_{N N}}=200 \mathrm{GeV}$, and 1000 collision events are generated.

The centrality of the heavy ion collision event is defined as $c=1-\frac{N_{\text {participants }}}{N_{\text {total }}}, N_{\text {total }}=A_{\text {projectile }}+$ $A_{\text {target }}=2 \times 197$ for ${ }^{197} A u_{79}$. Figure 1 shows the scatter graphs of the absolute value of the impact parameter $|\vec{b}|$ in Fermi units (fm) and the centrality of $A u-A u$ collision as produced by HIJING 1.35 in graph (1-a) and HYDJET ++2.0 .2 in graph (1-b). The monotonically decreasing strong correlation between $c$ and $|\vec{b}|$ is confirmed by Gaussian fitting to the most probable values. The illustration in Fig. 2 is the geometry of a single collision event. It represents the reaction plane in red as the plane containing both the impact parameter vector $\vec{b}$ and the incidence direction of both heavy ions.

The present work studies the asymmetry in antihadron to hadron production ratios concerning four selected hadrons in the reaction plane. Two mesons: $\pi^{ \pm}$as a low energy scalar meson and $\kappa^{ \pm}$as a strange meson, and two baryons: $p^{ \pm}$the protons and $\Lambda^{ \pm}$the lambda strange baryon, are selected in our study. The investigation of their multiplicity distributions are commonly reported [9].

The asymmetry sum is defined as,

$$
\begin{aligned}
\zeta= & \Sigma_{\text {events }} \frac{(\text { No. Antiparticles }- \text { No.particles })}{(\text { No. Antiparticles }+ \text { No.particles })} \\
& -N_{\text {events }} \leq \zeta \leq N_{\text {events }}
\end{aligned}
$$

\section{Results and discussions}

The momentum and space distributions of the hadron for the collision events produced by the HIJIJNG 1.35 event generator are obtained and presented in Fig. 3 for global antihadron to hadron ratios, and Fig. 4 for local asymmetry sum $\zeta$. In general there is a fault in the Hijing 1.35 hadronization mechanism for producing lambda baryons. The results for lambda particles are not trusted quantitatively, but the qualitative behavior is concerned as it is consistent with the validation made by V. V. Uzhinsky [9].

The symmetry in antihadron to hadron productions is balanced, if the ratios are equal to one and the asymmetry sums, $\zeta$, equals zero over all the space and momenta.

The distributions in Fig. 3 show, in general, larger values of antihadron to hadron ratios for $\pi^{ \pm}$, than for $\kappa^{ \pm}$ and finally for $p^{ \pm}$as $p^{+}$already exist in the colliding heavy ions, respectively. That is, the production of the anti-partner of the lightest meson is a candidate to test the HIJING model ability to produce the asymmetry in antihadron-hadron production [14]. Except for statistical fluctuations, the in reaction plane transverse momentum $P_{T_{i n}}$ behaviors are semi-decreasing $P_{T_{i n}}$ values. As shown in graph (3-Left) for HIJING 1.35 and graph (3-Right) for $H Y D J E T++$ 2.0.2. Kharzeev models also predict the enhancement in the production of anti-baryons with rapidity $y[4]$.

Figures (4-left) and (4-right) shows asymmetry ratios sum $\zeta$ distributions with $P_{T_{i n}}$ for baryons $p^{ \pm}$and $\Lambda^{ \pm}$. The mesons $\pi^{ \pm}$and $\kappa^{ \pm}$are symmetrical in the production of anti-hadrons. The anti-kaon production is enhanced in the 

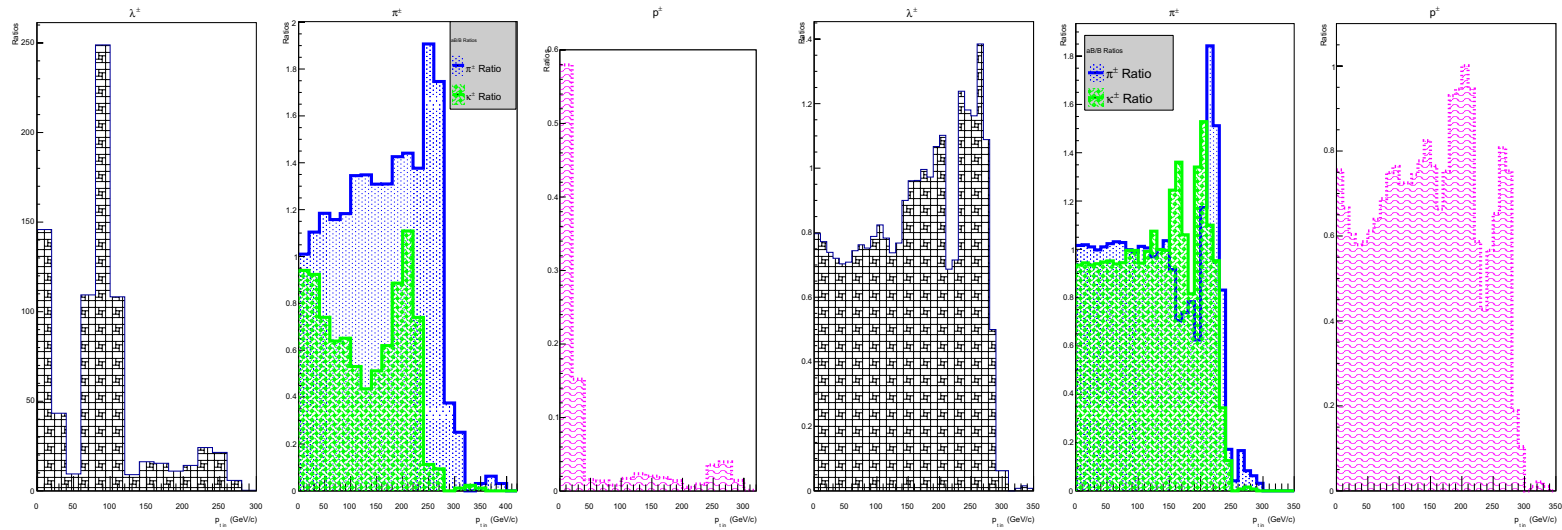

Figure 3. The $a h / h$ ratios in relation with $p_{T_{i n}}$ for mesons $\pi^{ \pm}$(blue), $\kappa^{ \pm}$(green)), and baryons ( $P^{ \pm}$(pink), $\Lambda^{ \pm}$(black)); (Left) HIJING 1.35. (Right) HYDJET++ 2.0.2.
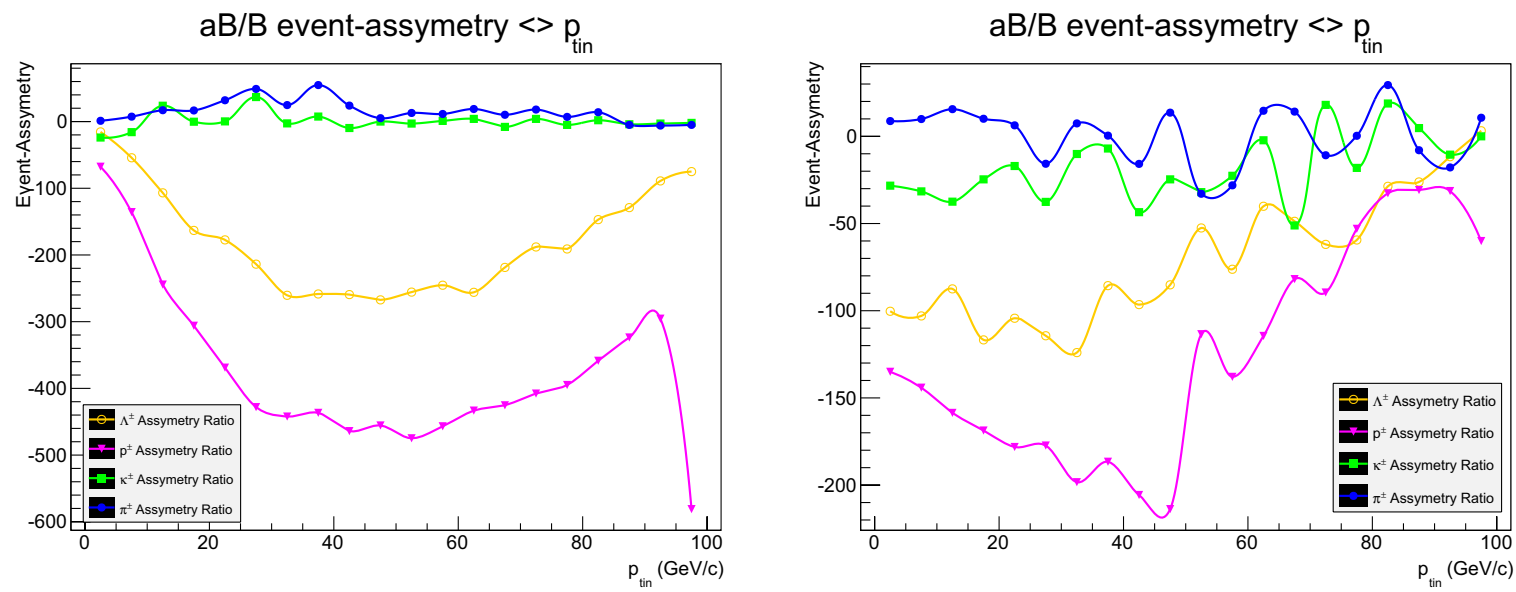

Figure 4. $a h / h$ event asymmetry ratio sum with respect to in reaction plane momentum $p_{T_{i n}}$ using (Left) HIJING 1.35 and (Right) HYDJET + +2.0 .2 simulator.
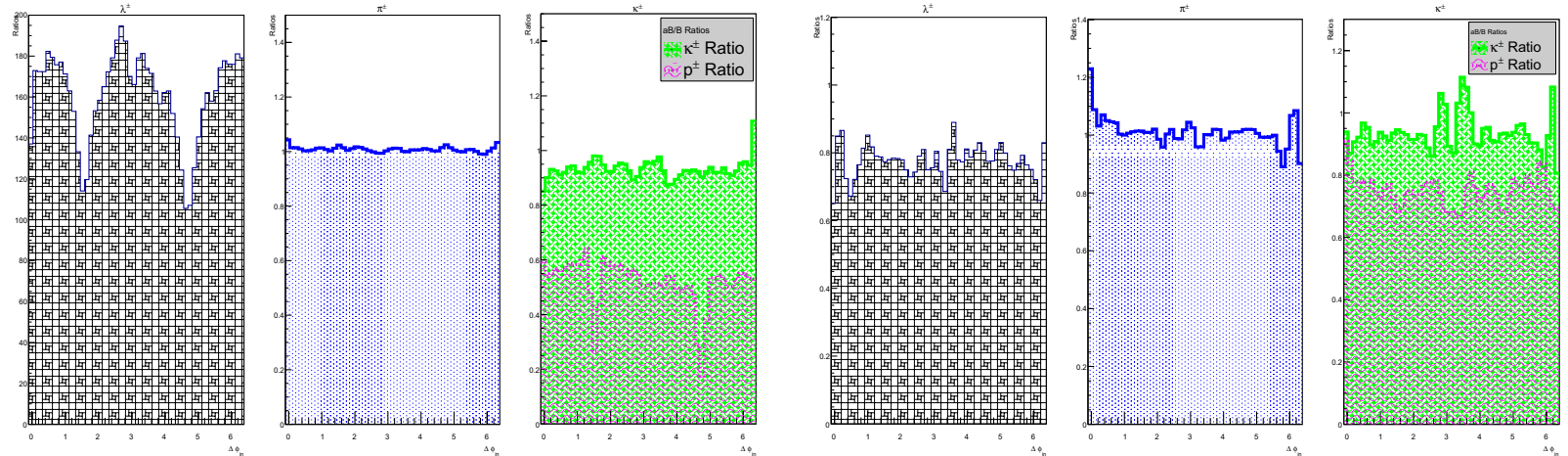

Figure 5. The $a h / h$ ratios in relation with $\Delta \phi_{i n}$ for mesons $\pi^{ \pm}$(blue), $\kappa^{ \pm}$(green)), and baryons ( $P^{ \pm}$(pink), $\Lambda^{ \pm}$(black)); (Left) HIJING 1.35. (Right) $H Y D J E T++2.0 .2$.

backward hemisphere, but the anti-proton production is enhanced in the forward hemisphere.

Figures (5-left) and (5-right) are the ratio distributions for the four particles with the azimuthal angle referenced with respect to the reaction plane (= direction of the impact parameter vector $|\vec{b}|$,) $\Delta \phi=\phi-\psi$, and the in-reaction plane azimuthal angle, $\Delta \phi_{i n}$. The distributions are symmetrical except for the proton $p^{ \pm}$ratio distribution for $\Delta \phi_{i n}$, The production of anti-protons is equally probable except for the angles $\Delta \phi_{i n}=\pi / 2,3 \pi / 2$ radians.
Figures (6-left) and (6-right) are the asymmetry ratio sum $\zeta$ for $\Delta \phi_{i n}=\phi_{i n}$. Irrespective of $p^{ \pm}$the production of particles and anti particles are uniform over the angles $\Delta \phi_{i n}=\phi_{i n}$, while for protons, $p^{ \pm}$, the protons production exceeds the anti-proton production at the angles $\Delta \phi_{i n}=$ $\pi / 2,3 \pi / 2$ radians.

\section{Conclusions}

HIJING 1.35 could reproduce the asymmetry in baryons and anti-baryons productions, but not for meson and 

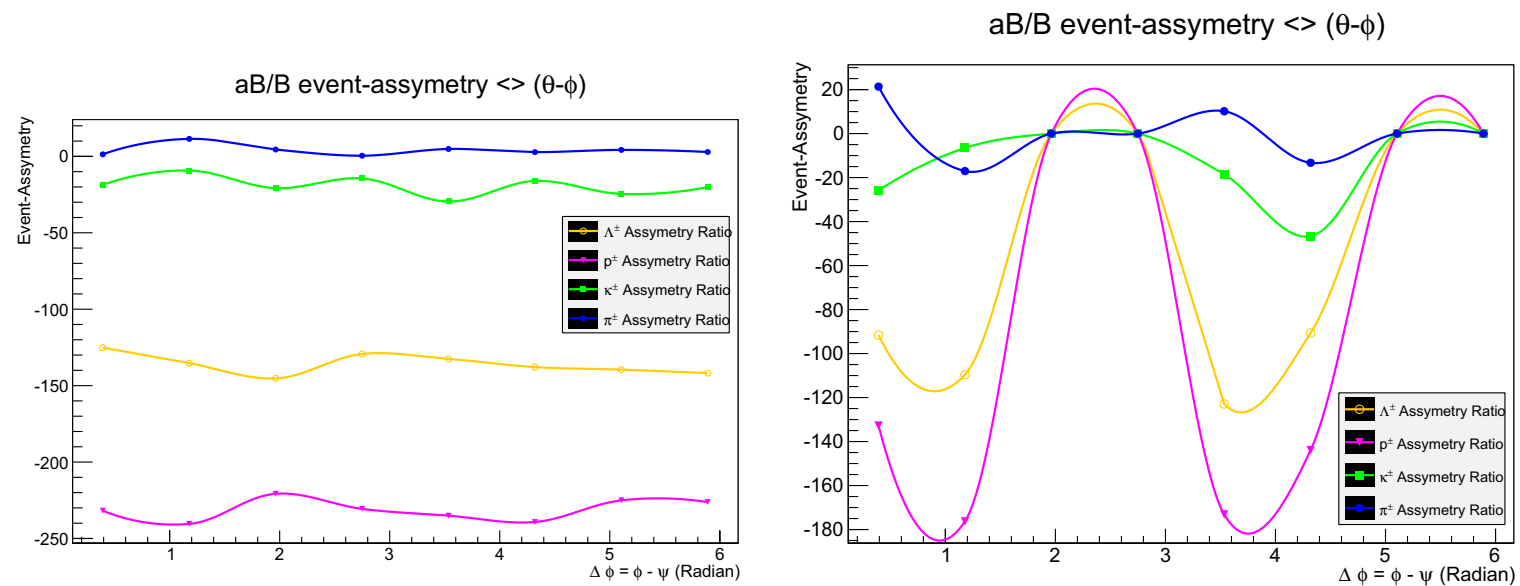

Figure 6. $a h / h$ event asymmetry ratio sum with respect to in reaction plane azimuthal angle $\Delta \phi_{i n}$ using (Left) HIJING 1.35 and (Right) HYDJET + + 2.0.2 simulator.

anti-mesons. The new defined parameter of asymmetry sum is statistically stable, especially, for baryons (which have larger masses than mesons), and shows a general asymmetry for baryons but not for mesons. The proton and anti-proton production according to the HIJING model shows a deficiency in anti-proton to proton production in the directions normal to the reaction plane, HYDJET ++ 2.0.2. The asymmetry ratio $\xi$ with $p_{T_{i n}}$ is decaying for $p^{ \pm}$in HIJING 1.35 and semi decaying for $p^{ \pm}$and $\Lambda^{ \pm}$in HYDJET ++ 2.0.2. HYDJET ++2.0 .2 predicts that the production of anti-baryons is enhanced with the $p_{T_{i n}}$ value increasing. For the in-reaction plane azimuthal angle $\Delta \phi_{i n}$, it is found that the asymmetry ratio sum $\zeta$ for the studied hadrons produced in events generated by HYDJET ++2.0 .2 in the interval $2 \leq$ $\Delta \phi_{\text {in }} \leq 4 \mathrm{rad}$ appears to decrease with some fluctuations. HYDJET ++2.0 .2 has been shown to be more realistic than HIJIN 1.35 in the study of dynamical parameters $p_{T_{i n}}$. It could be considered that the behavior of the asymmetry ratio sum $\zeta$ is a good representation of what can be obtained from the experimental measurements. The asymmetry in the distributions of charged particles (i. e. hadrons) could be observed very effectively using the statistically stable parameter of the asymmetry ratio sum, $\zeta$.

In future we intend to enter the effect of Kharzeev of the induced chiral magnetic field and the charge separation.

Thanks are due to Cairo University's research committee for partial funding of attendance at the symposium and encouraging the publishing of this work.

\section{References}

[1] STAR Collaboration, J. Adams, et al., Nucl. Phys. A 757, 102 (2005)

[2] STAR Collaboration, B. I. Abelev, et al., Nature 473, 353 (2011)

[3] Xin-Nian Wang, Miklos Gyulassy, Phys. Rev. D 44, 3501 (1991), Comput. Phys. Commun. 83, 307 (1994)

[4] Umut Gürsoy, Dmitri Kharzeev, Krishna Rajagopal, Nuclear Physics A 931, 986-991(2014)

[5] D.E. Kharzeev, et al., Nuclear Physics A 803, 227-253 (2008)

[6] S. A. Voloshin and A. M. Poskanzer, Phys. Lett. B 474, 27-32 (2000)

[7] STAR Collaboration, B. I. Abelev, et al., Phys. Rev. C 76, 024915 (2007)

[8] Jian-Hua Gao, Shou-Wan Chen, et al., Phys. Rev. C 77, $044902(2008)$

[9] V. V. Uzhinsky, arXiv: hep-Ph/0312089v2 (2003)

[10] I.P. Lokhtin, L.V. Malinina, S.V. Petrushanko, A.M. Snigirev, I. Arsene and K. Tywoniuk, Compu Phys. Commun. 180(5), 779-799 (2009)

[11] I.P. Lokhtin, L.V. Malinina, S.V. Petrushanko, A.M. Snigirev, I. Arsene and K. Tywoniuk, Compu. Phys. Commun. 180(5), 779-799 (2009)

[12] R. Brun and F. Rademakers, Nucl. Instrum. Meth. A 389, 81 (1997), http://root.cern.ch

[13] Igor.Lokhtin, http://cern.ch/lokhtin/ hydjet++, Last update: November 30 (2015) by Igor.Lokhtin@cern.ch

[14] AlLICE Collaboration, Abelev, and B. Bezverkhny, et al., Phys. Lett. B 728, 25-38 (2014), arXiv: nucl-ex/1307.6796v2, CERN-PH-EP-2013-135 